ANNALES

POLONICI MATHEMATICI

LXXIX.2 (2002)

\title{
Proper holomorphic self-mappings of the minimal ball
}

\author{
by NABIL OURIMi (Bizerte)
}

\begin{abstract}
The purpose of this paper is to prove that proper holomorphic self-mappings of the minimal ball are biholomorphic. The proof uses the scaling technique applied at a singular point and relies on the fact that a proper holomorphic mapping $f: D \rightarrow \Omega$ with branch locus $V_{f}$ is factored by automorphisms if and only if $f_{*}\left(\pi_{1}\left(D \backslash f^{-1}\left(f\left(V_{f}\right)\right), x\right)\right)$ is a normal subgroup of $\pi_{1}\left(\Omega \backslash f\left(V_{f}\right), b\right)$ for some $b \in \Omega \backslash f\left(V_{f}\right)$ and $x \in f^{-1}(b)$.
\end{abstract}

1. Introduction. Families of proper holomorphic mappings arise in the problem of determining which domains in $\mathbb{C}^{n}$ do not possess any proper holomorphic mappings which are not biholomorphic. In this paper our aim is to study this problem in the case of a special domain in $\mathbb{C}^{n}, n \geq 2$, with non-piecewise smooth boundary. This domain is the minimal ball. It is given by

$$
B_{\infty}=\left\{z \in \mathbb{C}^{n}: N_{\infty}(z)<1\right\},
$$

where $N_{\infty}(z)=\left(|z|^{2}+\left|z^{2}\right|\right) / 2$ and $z^{2}=\sum_{1 \leq j \leq n} z_{j}^{2}$.

The function $\sqrt{N_{\infty}}$ is a norm in $\mathbb{C}^{n}$ introduced by Hahn-Pflug [4] as the smallest norm in $\mathbb{C}^{n}$ that extends the Euclidean norm in $\mathbb{R}^{n}$ under certain restrictions. It has been studied in several recent works [7], [12], [9], [10], [11], [17]. The automorphism group of $B_{\infty}$ is $S^{1} . O(n, \mathbb{R})$ (see [7]). In addition $B_{\infty}$ is a non-Lu Qi-Keng domain for $n \geq 4$ and it is neither homogeneous nor Reinhardt. Its boundary is $B$-regular in the sense of Sibony [16] and Henkin-Iordan [6].

Our main result can be stated as follows:

THEOREM 1. Every proper holomorphic self-mapping of $B_{\infty}$ is biholomorphic.

2000 Mathematics Subject Classification: Primary 32H35.

Key words and phrases: proper holomorphic mappings, correspondences, branch locus, scaling, factorization, minimal ball. 
The following example shows that this theorem cannot be extended to proper holomorphic self-correspondences as in the case of strongly pseudoconvex domains (see [1]).

Let $\mathbb{M}=\left\{z \in \mathbb{C}^{n+1}:|z|<\sqrt{2}\right.$ and $\left.z^{2}=0\right\}$. The group $S^{1} . O(n+1, \mathbb{R})$ is a subgroup of $\operatorname{Aut}(\mathbb{M})$. Consider the projection pr : $\mathbb{C}^{n+1} \rightarrow \mathbb{C}^{n}$ defined by $\operatorname{pr}\left(z_{1}, \ldots, z_{n+1}\right)=\left(z_{1}, \ldots, z_{n}\right)$. The restriction $F:=\operatorname{pr} \mid \mathbb{M}$ is a proper holomorphic mapping with multiplicity 2 from $\mathbb{M}$ onto $B_{\infty} \subset \mathbb{C}^{n}$. Let $g \in$ $S^{1} . O(n+1, \mathbb{R}) \backslash S^{1} . O(n, \mathbb{R})$ (the group $O(n, \mathbb{R})$ can be regarded as a subgroup of $O(n+1, \mathbb{R}))$. Then $h=F \circ g \circ F^{-1}$ is an irreducible proper holomorphic self-correspondence of $B_{\infty}$. To prove that $h$ is a nontrivial correspondence, assume that $h$ is a mapping. Then $h$ is an automorphism of $B_{\infty}$ (i.e. $h \in$ $S^{1} . O(n, \mathbb{R})$ ); otherwise the multiplicity of $h \circ F$ will be greater than the multiplicity of $F \circ g$. This implies that $g \in S^{1} . O(n, \mathbb{R})$ and so we get a contradiction.

2. Preliminary results. In this section, we give some preliminary results useful for the proof of our theorem.

2.1. Factorization of proper holomorphic mappings. A mapping $f: D \rightarrow$ $\Omega$ is factored by automorphisms if there is a finite subgroup $\Gamma \subset \operatorname{Aut}(D)$ such that for all $z$ in $D$,

$$
f^{-1}(f(z))=\{\gamma(z): \gamma \in \Gamma\}
$$

We will denote by $J_{f}(z)$ the Jacobian determinant of $f$ and by $V_{f}=$ $\left\{z \in D: J_{f}(z)=0\right\}$ its branch locus. A necessary and sufficient condition to factorize proper holomorphic mappings is given in the following theorem.

Theorem 2. Let $D$ be a pseudoconvex bounded domain in $\mathbb{C}^{n}, \Omega$ a domain in $\mathbb{C}^{n}$ and $f: D \rightarrow \Omega$ a proper holomorphic mapping with branch locus $V_{f}$. Denote by $F$ the restriction of $f$ to $D \backslash f^{-1}\left(f\left(V_{f}\right)\right)$. Then the following statement are equivalent:

(1) there exist $b \in \Omega \backslash f\left(V_{f}\right)$ and $x \in f^{-1}(b)$ such that $F_{*}\left(\pi_{1}(D \backslash\right.$ $\left.\left.f^{-1}\left(f\left(V_{f}\right)\right), x\right)\right)$ is a normal subgroup of $\pi_{1}\left(\Omega \backslash f\left(V_{f}\right), b\right)$,

(2) $f$ is factored by automorphisms.

The existence of the group $\Gamma$ is due to W. Rudin [15] in the case of the Euclidean ball in $\mathbb{C}^{n}$ and to Bedford-Bell [1] in the case of strongly pseudoconvex domains in $\mathbb{C}^{n}$ (see also the references for related results). Theorem 2 implies that the branch locus of $f$ is given by

$$
V_{f}=\bigcup_{\{g \in \Gamma: g \neq \mathrm{id}\}}\{z \in D: g(z)=z\} .
$$

Then the factorization theorem above may be used to reduce the study of the behavior of the branch locus to the study of the group $\Gamma$. Thus far $\Gamma$ has been identified only in the case of the Euclidean ball in $\mathbb{C}^{n}$. 
Proof of Theorem 2. Let $H=f\left(V_{f}\right), E=D \backslash f^{-1}(H)$ and $B=\Omega \backslash H$. The restriction $F=f \mid E: E \rightarrow B$ is a connected finite covering. According to [3], $F_{*}\left(\pi_{1}(E, x)\right)$ is a normal subgroup in $\pi_{1}(B, b)$ if and only if $F$ : $E \rightarrow B$ is a Galois covering, i.e. $F$ is a connected covering and the group $\Gamma=\{\gamma \in \operatorname{Hom}(E): F \circ \gamma=F\}$ acts transitively on each fiber $F^{-1}(b)$, $b \in B$. Moreover, the group $\Gamma$ is isomorphic to $\pi_{1}(B, b) / F_{*}\left(\pi_{1}(E, x)\right)$.

Assume that $F_{*}\left(\pi_{1}(E, x)\right)$ is a normal subgroup in $\pi_{1}(B, b)$. Then $F$ is a Galois covering. The mapping $F$ is holomorphic, so all elements of $\Gamma$ are biholomorphic and since $D$ is bounded, they extend to holomorphic mappings from $D$ onto $\bar{D}$. Suppose that there exists a point $p \in f^{-1}(H)$ such that $\gamma(p) \in \partial D$ for some $\gamma \in \Gamma$. Let $\Delta$ be an analytic disc in $D$ such that $\Delta \cap f^{-1}(H)=\{p\}$. Then $\gamma(\Delta)$ intersects $\partial D$ only at $\gamma(p)$. This contradicts the fact that $D$ is pseudoconvex. Hence for all $\gamma \in \Gamma, \gamma(D) \subset D$. Thus the elements of $\Gamma$ extend to automorphisms of $D$ and define a subgroup of $\operatorname{Aut}(D)$ that we denote by $\widehat{\Gamma}$. By analytic extension the equality $f \circ \widehat{\gamma}=f$ remains valid for all $\widehat{\gamma} \in \widehat{\Gamma}$.

Let now $b \in H$ and $z_{1}, z_{2} \in f^{-1}(H)$ be such that $f\left(z_{1}\right)=f\left(z_{2}\right)=b$. Since $f$ is an open map, there exist two sequences $\left\{z_{1}^{j}\right\}_{j}$ and $\left\{z_{2}^{j}\right\}_{j}$ in $E$ that converge respectively to $z_{1}$ and $z_{2}$ and satisfy $f\left(z_{1}^{j}\right)=f\left(z_{2}^{j}\right)$ for all $j$. The mapping $F$ is a Galois covering, so for all $j$ there exists $\gamma_{j} \in \Gamma$ such that $z_{1}^{j}=\gamma_{j}\left(z_{2}^{j}\right)$. As $\Gamma$ is a finite subgroup, we may assume that $z_{1}^{j}=\gamma\left(z_{2}^{j}\right)$ for some $\gamma \in \Gamma$ and for any integer $j$. Passing to the limit, we get $z_{1}=\widehat{\gamma}\left(z_{2}\right)$ $(\widehat{\gamma}$ is the extension of $\gamma$ ). This proves that the mapping $f$ is factored by $\widehat{\Gamma}$.

Conversely, assume that $f$ is factored by a subgroup $\widehat{\Gamma}$. It is clear that for all $\widehat{\gamma} \in \widehat{\Gamma}, \widehat{\gamma}$ maps $E$ onto itself. Then the restriction $F$ is a Galois covering. This implies that $F_{*}\left(\pi_{1}(E, x)\right)$ is a normal group in $\pi_{1}(B, b)$.

2.2. Hopf's lemma for $B_{\infty}$. We denote by $\varrho=\sqrt{N_{\infty}}-1$ a defining function of $B_{\infty}$. It is easy to see that this function satisfies the following lemma.

Lemma 1. For $z \in B_{\infty}$ one has

$$
\frac{1}{\sqrt{2}} \operatorname{dist}\left(z, \partial B_{\infty}\right) \leq|\varrho(z)| \leq \operatorname{dist}\left(z, \partial B_{\infty}\right) .
$$

First, we establish the uniform Hopf lemma for the unit disc $\Delta$ in $\mathbb{C}$.

Lemma 2. Let $r$ be a subharmonic negative function on $\Delta$. Then for all $z \in \Delta$ one has

$$
|r(z)| \geq \inf _{\Delta(0,1 / 2)}|r| \operatorname{dist}(z, \partial \Delta) .
$$

Proof. We consider the subharmonic function

$$
\widehat{r}(z)=r(z)-A \frac{\operatorname{Ln}|z|}{\operatorname{Ln} 2}, \quad \text { where } \quad A=\inf _{\bar{\Delta}(0,1 / 2)}|r| .
$$


Since $\widehat{r}$ is negative on $\partial \Delta(0,1 / 2)$ and $\overline{\lim }_{z \rightarrow z_{0} \in \partial \Delta} \widehat{r}(z) \leq 0$, by the maximum principle we get $\widehat{r}(z) \leq 0$ on $\Delta(0,1) \backslash \Delta(0,1 / 2)$. Thus

$$
\forall z \in \Delta, \quad|r(z)| \geq \inf _{\bar{\Delta}(0,1 / 2)}|r| \min \left(1, \frac{-\operatorname{Ln}|z|}{\operatorname{Ln} 2}\right) \geq \inf _{\bar{\Delta}(0,1 / 2)}|r|(1-|z|) .
$$

As an application, we get the Hopf lemma for the minimal ball.

Lemma 3. Let $r$ be a plurisubharmonic negative function on $B_{\infty}$. Then for all $z \in B_{\infty}$ one has

$$
|r(z)| \geq \frac{1}{\sqrt{2}} \inf _{\frac{1}{2} \bar{B}_{\infty}}|r| \operatorname{dist}\left(z, \partial B_{\infty}\right),
$$

where $\frac{1}{2} \bar{B}_{\infty}=\left\{z \in \mathbb{C}^{n}: \sqrt{N_{\infty}}(z) \leq 1 / 2\right\}$.

Proof. For $z=0$ the inequality is true. Let now $z \in B_{\infty} \backslash\{0\}$ and consider the subharmonic function $u$ defined on the unit disc by

$$
u(\xi)=r\left(\frac{\xi z}{\sqrt{N_{\infty}}(z)}\right) .
$$

In view of Lemma 2 we have

$$
|u(\xi)| \geq \inf _{\bar{\Delta}(0,1 / 2)}|u| \operatorname{dist}(\xi, \partial \Delta) .
$$

Let $\xi=\sqrt{N_{\infty}}(z)$. The previous inequality becomes

$$
|r(z)| \geq \inf _{\Delta(0,1 / 2)}|u| \cdot\left|1-\sqrt{N_{\infty}}(z)\right| \geq \frac{1}{\sqrt{2}} \inf _{\bar{\Delta}(0,1 / 2)}|u| \operatorname{dist}\left(z, \partial B_{\infty}\right) .
$$

Since

$$
\inf _{w \in \bar{\Delta}(0,1 / 2)}\left|r\left(\frac{w z}{\sqrt{N_{\infty}}(z)}\right)\right| \geq \inf _{\frac{1}{2} \bar{B} \infty_{\infty}}|r|
$$

we have the desired inequality.

In the case $n=2$, the minimal ball is biholomorphic to the Reinhardt domain $\left\{(z, w) \in \mathbb{C}^{2}:|z|+|w|<1\right\}$. Hence according to [2], any proper holomorphic self-mapping of $B_{\infty}$ is biholomorphic. Now assume that $n \geq 3$. The proof of our theorem is based on the scaling technique and the notion of factorization of proper holomorphic mappings.

3. Scaling technique. Set $H=\left\{z \in \mathbb{C}^{n}: \sum_{1 \leq j \leq n} z_{j}^{2}=0\right\}$ and $H_{\infty}=H \cap B_{\infty}$. The singular part of the boundary of $B_{\infty}$ is obviously the set $\partial H_{\infty}=H \cap \partial B_{\infty}$. The regular part $\partial B_{\infty} \backslash \partial H_{\infty}$ is $C^{\infty}$-smooth and it consists of strongly pseudoconvex points. In [10] the authors gave an explicit formula for the Bergman kernel of the minimal ball. In particular, they showed that any proper holomorphic self-mapping of the minimal ball 
extends holomorphically to a neighborhood of $\bar{B}_{\infty}$ (see Theorem 5.5 of [10]). In this section we shall prove the following:

Proposition 1. Let $f$ be a branching proper holomorphic self-mapping of $B_{\infty}$ with branch locus $V_{f}$. Then $f\left(V_{f}\right)$ is equal to $H_{\infty}$.

Proof. Let $p \in \partial V_{f}=\bar{V}_{f} \backslash V_{f}$ and let $\left\{p_{k}\right\}_{k}$ be a sequence in $V_{f}$ that converges to $p$. Since $f$ is proper, the sequence $\left\{f\left(p_{k}\right)\right\}_{k}$ converges to a boundary point $q \in \partial B_{\infty}$. We shall prove that $q$ is a singular point (i.e. $q \in \partial H_{\infty}$ ). The proof is by contradiction. Assume that $q$ is a strong pseudoconvexity point. We will discuss two cases.

First case: $p$ is a strong pseudoconvexity point. Since the mapping $f$ extends holomorphically to a neighborhood of $\bar{B}_{\infty}([10])$, it defines a local biholomorphism near $p$ (see [14]). This contradicts the fact that $p \in \partial V_{f}$.

Second case: $p$ is a singular point $\left(p \in \partial H_{\infty}\right)$. In this case we will use the scaling technique to prove that this situation is not possible.

Since $S^{1} . O(n, \mathbb{R})$ acts transitively on $\partial H_{\infty}$, we can assume without loss of generality that $p=(0, \ldots, 0, i, 1)$. The domain $B_{\infty}-p$ is represented by $2 \operatorname{Re}\left(z_{n}-i z_{n-1}\right)+\left|z_{1}\right|^{2}+\ldots+\left|z_{n}\right|^{2}+\left|z_{1}^{2}+\ldots+z_{n}^{2}+2\left(z_{n}+i z_{n-1}\right)\right|<0$.

In the new coordinates

$$
\begin{aligned}
\zeta_{j} & =z_{j}, \quad j \in\{1, \ldots, n-2\}, \\
\zeta_{n-1} & =z_{n}+i z_{n-1}, \\
\zeta_{n} & =z_{n}-i z_{n-1},
\end{aligned}
$$

the point $p$ is transformed to 0 and the domain $B_{\infty}-p$ corresponds to the domain $G$ defined by $\{\varphi<0\}$ with

$$
\begin{aligned}
\varphi(\xi)= & 2 \operatorname{Re}\left(\zeta_{n}\right)+\frac{1}{2}\left(\left|\zeta_{n}\right|^{2}+\left|\zeta_{n-1}\right|^{2}\right)+\left|\zeta_{1}\right|^{2}+\ldots+\left|\zeta_{n-2}^{2}\right| \\
& +\left|\zeta_{1}^{2}+\ldots+\zeta_{n-2}^{2}+2 \zeta_{n-1}+\zeta_{n-1} \zeta_{n}\right| .
\end{aligned}
$$

We write $z \in \mathbb{C}^{n}$ as $z=\left({ }^{\prime} z, z_{n}\right)$ where ' $z$ denotes the first $n-1$ coordinates of $z$ or $z=\left({ }^{\prime \prime} z, z_{n-1}, z_{n}\right)$ where " $z$ denotes the first $n-2$ coordinates of $z$. If $\left\{a_{k}\right\}_{k}$ and $\left\{b_{k}\right\}_{k}$ are two sequences of real positive numbers, we write $a_{k} \simeq b_{k}$ if there is a positive constant $c$ independent of $k$ such that $c^{-1} b_{k} \leq a_{k} \leq c b_{k}$.

Let $g: B_{\infty} \rightarrow G$ be the linear transformation mapping $B_{\infty}$ onto $G$ and $t_{k}=\left({ }^{\prime} 0,-\delta_{k}\right), k=1,2, \ldots$, be a sequence of points in $G$ where $\left\{\delta_{k}\right\}_{k}$ is a sequence of real positive numbers converging to 0 . It is clear that for large $k$, $\left|\varphi\left(t_{k}\right)\right| \simeq \delta_{k}$. Since $B_{\infty}$ is linearly equivalent to $G$, in view of Lemma 1 we have, for all $z \in G$,

$$
\frac{1}{\sqrt{2}\|g\|} \operatorname{dist}(z, \partial G) \leq|\varphi(z)| \leq\left\|g^{-1}\right\| \operatorname{dist}(z, \partial G) .
$$


It follows that for large $k$,

$$
\operatorname{dist}\left(t_{k}, \partial G\right) \simeq \delta_{k} .
$$

As the mapping $f$ is continuous, the sequence $\left\{q_{k}\right\}_{k}, q_{k}=f \circ g^{-1}\left(t_{k}\right)$, converges to $q=f \circ g^{-1}(0)$. Let $V$ be a neighborhood of $q$ in $\mathbb{C}^{n}$ which does not intersect the set of weakly pseudoconvex points of $\partial B_{\infty}$. For all $w \in \partial B_{\infty} \cap V$ we consider the change of variables $h^{w}$ defined by

$$
\begin{aligned}
z_{j}^{*} & =\frac{\partial \varrho}{\partial \bar{z}_{n}}(w)\left(z_{j}-w_{j}\right)-\frac{\partial \varrho}{\partial \bar{z}_{j}}(w)\left(z_{n}-w_{n}\right), \quad 1 \leq j \leq n-1, \\
z_{n}^{*} & =\sum_{1 \leq j \leq n} \frac{\partial \varrho}{\partial z_{j}}(w)\left(z_{j}-w_{j}\right) .
\end{aligned}
$$

The mapping $h^{w}$ maps $w$ to 0 and the real normal to $\partial B_{\infty}$ at $w$ onto the line $\left\{{ }^{\prime} z=0, y_{n}=0\right\}$. Let $w_{k}$ be the projection of $q_{k}$ on the boundary of $B_{\infty}$. For simplicity we denote $h^{w_{k}}$ (the mapping as above) by $h^{k}$. Set $D^{k}=h^{k}\left(B_{\infty}\right)$, $\varrho^{k}=\varrho \circ h^{k^{-1}}$ and $\gamma_{k}=\operatorname{dist}\left(h^{k}\left(q_{k}\right), \partial D^{k}\right)$. We have $h^{k}\left(q_{k}\right)=\left({ }^{\prime} 0,-\gamma_{k}\right)$. The sequence of proper holomorphic mappings defined by $f^{k}=h^{k} \circ f \circ g^{-1}$ : $G \rightarrow D^{k}$ satisfies $f^{k}\left({ }^{\prime} 0,-\delta_{k}\right)=\left({ }^{\prime} 0,-\gamma_{k}\right)$ for all $k$. Now we introduce the inhomogeneous dilatation of coordinates as follows:

$$
\alpha^{k}\left({ }^{\prime} z, z_{n}\right)=\left(\frac{{ }^{\prime} z}{\sqrt{\gamma}_{k}}, \frac{z_{n}}{\gamma_{k}}\right), \quad \beta^{k}\left({ }^{\prime \prime} z, z_{n-1}, z_{n}\right)=\left(\frac{{ }^{\prime \prime} z}{\sqrt{\delta}_{k}}, \frac{z_{n-1}}{\delta_{k}}, \frac{z_{n}}{\delta_{k}}\right) .
$$

The idea is to follow the argument of Pinchuk [13] and to consider the mapping $\widehat{f}^{k}=\alpha^{k} \circ f^{k} \circ\left(\beta^{k}\right)^{-1}$. In the new coordinates, $G$ and $D^{k}$ correspond to the domains $\widehat{G}^{k}$ and $\widehat{D}^{k}$ with defining functions

$$
\widehat{\varphi}^{k}(z)=\frac{1}{\delta_{k}} \varphi \circ \beta^{k^{-1}}(z), \quad \varrho^{k}(z)=\frac{1}{\gamma_{k}} \varrho^{k} \circ\left(\alpha^{k}\right)^{-1}(z)
$$

respectively. Thus, $\widehat{f}^{k}$ is a proper holomorphic mapping from $\widehat{G}^{k}$ onto $\widehat{D}^{k}$ and satisfies $\widehat{f}^{k}\left({ }^{\prime} 0,-1\right)=\left({ }^{\prime} 0,-1\right)$. Let $\widehat{G}=\{\widehat{\varphi}<0\}$ and $\Sigma=\{\widehat{\varrho}<0\}$ where $\widehat{\varphi}(z)=2 \operatorname{Re}\left(z_{n}\right)+\left|{ }^{\prime \prime} z\right|^{2}+\left.\right|^{\prime \prime} z^{2}+\left.2 z_{n-1}\left|, \widehat{\varrho}(z)=2 \operatorname{Re}\left(z_{n}\right)+\right|{ }^{\prime} z\right|^{2}$ and ${ }^{\prime \prime} z^{2}=\sum_{1 \leq j \leq n-2} z_{j}^{2}$. The sequence $\left\{\widehat{\varphi}^{k}\right\}_{k}$ (resp. $\left\{\varrho^{k}\right\}_{k}$ ) converges uniformly to the function $\widehat{\varphi}$ on compact subsets of $\widehat{G}$ (resp. to the function $\widehat{\varrho}$ on compact subsets of $\Sigma$ ). Consequently, for all compact $K \subset \widehat{G}$, the mappings $\widehat{f}^{k}$ are well defined on $K$, starting from some $k^{0}=k^{0}(K)$. By exhausting $\widehat{G}$ with an increasing sequence of compact sets and by passing to the limit, we conclude that we may assume that $\left\{\widehat{f}^{k}\right\}_{k}$ converges to a holomorphic function $\widehat{f}: \widehat{G} \rightarrow \bar{\Sigma}$. Since $\widehat{\varrho}$ is plurisubharmonic and $\widehat{f}\left({ }^{\prime} 0,-1\right)=\left({ }^{\prime} 0,-1\right)$ $\in \Sigma$, the maximum principle implies that $\widehat{f}(\widehat{G}) \subset \Sigma$.

We shall prove that $\widehat{f}$ is proper. For this we need some estimates on the distance. 
Lemma 4. There exists a constant $c>0$ such that for all $z \in B_{\infty}$,

$$
\frac{1}{c} \operatorname{dist}\left(z, \partial B_{\infty}\right) \leq \operatorname{dist}\left(f(z), \partial B_{\infty}\right) \leq c \operatorname{dist}\left(z, \partial B_{\infty}\right) .
$$

Proof. Recall that $\varrho$ denotes a defining function of $B_{\infty}$. Since $\varrho \circ f$ is plurisubharmonic and negative on $B_{\infty}$, in view of Lemmas 1 and 3 there exists a constant $c>0$ such that for all $z \in B_{\infty}, \operatorname{dist}\left(f(z), \partial B_{\infty}\right) \geq$ $c^{-1} \operatorname{dist}\left(z, \partial B_{\infty}\right)$. To prove the right-hand inequality, we consider the function $r(w)=\max \left\{\varrho(z): z \in f^{-1}(w)\right\}$, which is well defined and plurisubharmonic on $B_{\infty} \backslash f\left(V_{f}\right)$ and also bounded there. Since $f$ is proper, $f\left(V_{f}\right)$ is an analytic subvariety, and so $r$ extends as a plurisubharmonic function on $B_{\infty}$. Now we apply Lemmas 1 and 3 again.

Since the coordinates $h^{w_{k}}$ depend continuously on $w_{k}$ and the domain $G$ is linearly equivalent to $B_{\infty}$, in view of Lemma 4 the following estimates hold:

$$
c_{1} \operatorname{dist}(z, \partial G) \leq \operatorname{dist}\left(f^{k}(z), \partial D^{k}\right) \leq c_{2} \operatorname{dist}(z, \partial G),
$$

with $c_{1}, c_{2}>0$ do not depend on $k$. In addition, in $G$ and $D^{k}$ we have the estimates

$$
\begin{aligned}
c_{3}|\varphi(z)| & \leq \operatorname{dist}(z, \partial G) \leq c_{4}|\varphi(z)|, \\
c_{3}\left|\varrho^{k}(w)\right| & \leq \operatorname{dist}\left(w, \partial D^{k}\right) \leq c_{4}\left|\varrho^{k}(w)\right|,
\end{aligned}
$$

where $c_{3}, c_{4}>0$ do not depend on $k$ (the estimates (3) follow from Lemmas 1 and 3). According to (1) and (2), there exist positive constants $c_{5}$ and $c_{6}$ independent of $k$ such that for all $k$,

$$
c_{5}<\gamma_{k} / \delta_{k}<c_{6} \text {. }
$$

Let $K$ be a compact set in $\widehat{G}$ and $z \in K$. Set $w^{k}=\widehat{f}^{k}(z)$. In view of (2)-(4),

$$
\begin{aligned}
\varrho^{k}\left(w^{k}\right) & =\gamma_{k}{ }^{-1} \varrho^{k}\left(\sqrt{\gamma}_{k}{ }^{\prime} w^{k}, \gamma_{k} w_{n}^{k}\right) \leq\left(\gamma_{k} c_{3}\right)^{-1} \operatorname{dist}\left(\left(\sqrt{\gamma}_{k}{ }^{\prime} w^{k}, \gamma_{k} w_{n}^{k}\right), \partial D^{k}\right) \\
& \leq c_{2}\left(\gamma_{k} c_{3}\right)^{-1} \operatorname{dist}\left(\left(\sqrt{\delta}_{k}{ }^{\prime \prime} z, \delta_{k} z_{n-1}, \delta_{k} z_{n}\right), \partial G\right) \\
& \leq c_{2} c_{4}\left(\gamma_{k} c_{3}\right)^{-1} \varphi\left(\sqrt{\delta}_{k}{ }^{\prime \prime} z, \delta_{k} z_{n-1}, \delta_{k} z_{n}\right) \\
& =c_{2} c_{4} \delta_{k}\left(c_{3} \gamma_{k}\right)^{-1} \widehat{\varphi}^{k}(z) \leq c_{2} c_{4}\left(c_{5} c_{3}\right)^{-1} \widehat{\varphi}^{k}(z) .
\end{aligned}
$$

Passing to a convergent subsequence and to the limit, we get

$$
\widehat{\varrho}(\widehat{f}(z)) \leq c_{7} \widehat{\varphi}(z)
$$

for $z \in K$ and for some positive constant $c_{7}$ independent of $z$. Since $K$ is an arbitrary compact set in $\widehat{G}$, the estimate (5) holds for all $z \in \widehat{G}$.

Lemma 5. The sequence $\left\{\widehat{f}^{k}\right\}_{k}$ admits a subsequence converging uniformly on compact subsets of $\widehat{G}$ to a proper holomorphic mapping $\widehat{f}: \widehat{G} \rightarrow \Sigma$. 
Proof. The proof is based on certain ideas of S. Pinchuk [13]. For the convenience of the reader and for the sake of completeness we include a proof. We consider the function $v(z)=e^{z_{n}}$. It satisfies $|v(z)|<1$ on $\bar{G} \backslash\{0\}$ and $v(0)=1$. For $\delta_{k}<1$ the functions

$$
v^{k}(z)=\frac{v(z)-\left(1-\delta_{k}\right)}{1-v(z)\left(1-\delta_{k}\right)}
$$

are holomorphic in a neighborhood of $\bar{G}$ and $\left|v^{k}(z)\right|<1$ for all $z \in G$. For large $k$ we consider the function $u^{k}(z)=v^{k}\left(\sqrt{\delta}_{k}{ }^{\prime \prime} z, \delta_{k} z_{n-1}, \delta_{k} z_{n}\right)$, which is holomorphic in a neighborhood of $\widehat{G}^{k}$ and has the form

$$
u^{k}(z)=\frac{1+z_{n}+\delta_{k}\left(\sum_{p \geq 2} \frac{\delta_{k}^{p-2}}{p !} z_{n}^{p}\right)}{1-z_{n}\left(1-\delta_{k}\right)-\delta_{k}\left(\sum_{p \geq 2} \frac{\delta_{k}^{p-2}}{p !} z_{n}^{p}\right)\left(1-\delta_{k}\right)} .
$$

Set $u^{0}=\lim _{k \rightarrow \infty} u^{k}$. The limit $u^{0}$ is holomorphic on $\widehat{G}$ and it is defined by

$$
u^{0}(z)=\frac{1+z_{n}}{1-z_{n}} .
$$

As $|z| \rightarrow \infty$ and $z \in \widehat{G}$, clearly $\left|z_{n}\right| \rightarrow \infty$. Thus $u^{0}(z) \rightarrow-1$ as $|z| \rightarrow \infty$. Since the mappings $\widehat{f}^{k}: \widehat{G}^{k} \rightarrow \widehat{D}^{k}$ are ramified analytic coverings (see [5]), for each $k$ there exists a polynomial

$$
P^{k}(t, w)=t^{m}+S_{1}^{k}(w) t^{m-1}+\ldots+S_{m}^{k}(w)
$$

(where $S_{\alpha}^{k}$ are holomorphic functions on $\widehat{D}^{k}$ for $\alpha \in\{1, \ldots, m\}$ and $m$ is the multiplicity of $\left.\widehat{f}^{k}\right)$ such that $P^{k}\left(u^{k}(z), \widehat{f}^{k}(z)\right) \equiv 0$ on $\widehat{G}^{k}$ and for $w \in \widehat{D}^{k}$ one has $P^{k}(t, w)=0$ if and only if $t \in u^{k} \circ\left(\widehat{f}^{k}\right)^{-1}(w)$. As $\left|u^{k}(z)\right|<1$ in $\widehat{G}^{k}$, it follows that all the roots of $P^{k}(\cdot, w)$ are of modulus less than one for all $w \in \widehat{D}^{k}$. Then there exists a constant $c>0$ such that $\left|S_{\alpha}^{k}(w)\right|<c$ in $\widehat{D}^{k}$. Consequently, we can assume (after passing to a subsequence) that for all $\alpha \in\{1, \ldots, m\}$ the sequence $\left\{S_{\alpha}^{k}\right\}_{k}$ converges to a function $S_{\alpha}$, defined and holomorphic in $\Sigma$. Let $P(t, w)=t^{m}+S_{1}(w) t^{m-1}+\ldots+S_{m}(w)$. We have $P\left(u^{o}(z), \widehat{f}(z)\right) \equiv 0$ and the roots of $P$ lie in the closed unit disc for all $w \in \Sigma$. We write $P(t, w)$ in the form $(t+1)^{l} Q(t, w)$, where $Q(-1, w) \not \equiv 0$.

Assume that $\widehat{f}$ is not proper. In view of (5) there exists a sequence $\left\{z_{\mu}\right\}_{\mu}$ of $\widehat{G}$ such that $z_{\mu} \rightarrow \infty$ and $\widehat{f}\left(z_{\mu}\right) \rightarrow w^{0} \in \Sigma$ as $\mu \rightarrow \infty$. By the Weierstrass preparation theorem, $Q(t, w)$ can be written as $Q_{1}(t, w) \cdot Q_{2}(t, w)$ in a neighborhood of $\left(-1, w^{o}\right)$ where $Q_{1}\left(-1, w^{o}\right) \neq 0$ and

$$
Q_{2}(t, w)=\left(w_{n}-w_{n}^{o}\right)^{r}+a_{1}\left(t,{ }^{\prime} w\right)\left(w_{n}-w_{n}^{o}\right)^{r-1}+\ldots+a_{r}\left(t,{ }^{\prime} w\right) .
$$


If $t^{0}$ is close to -1 and $\left|t^{\circ}\right|>1$, the equation $Q_{2}(t, w)=0$ has a root $w^{1}$ in $\Sigma$ close to $w^{o}$, and so $P\left(t^{o}, w^{1}\right)=0$. This contradicts the fact that the roots of $P$ lie in the closure of the unit disc and proves that $\widehat{f}$ is proper.

We need the following proposition.

Proposition 2 ([13]). Let at least one of the domains $\Omega_{1}, \Omega_{2} \subset \subset \mathbb{C}^{n}$ be strongly pseudoconvex, and assume that there exists a proper holomorphic mapping from $\Omega_{1}$ onto $\Omega_{2}$ which is not biholomorphic. Then there is no proper holomorphic mapping from $\Omega_{2}$ onto $\Omega_{1}$. In particular $\Omega_{1}$ and $\Omega_{2}$ are biholomorphically inequivalent.

Conclusion of the proof of Proposition 1. It is clear that $\widehat{G}$ is biholomorphic to the domain $E_{1}=\left\{\left({ }^{\prime \prime} z, z_{n-1}, z_{n}\right) \in \mathbb{C}^{n}: 2 \operatorname{Re}\left(z_{n}\right)+\left.\left.\right|^{\prime \prime} z\right|^{2}+\left|z_{n-1}\right|<0\right\}$. The fractional transformation

$$
\left({ }^{\prime \prime} z, z_{n-1}, z_{n}\right) \mapsto\left(\frac{\sqrt{2}^{\prime \prime} z}{z_{n}-1}, \frac{2 z_{n-1}}{\left(z_{n}-1\right)^{2}}, \frac{z_{n}+1}{z_{n}-1}\right)
$$

maps $E_{1}$ biholomorphically onto the domain $E_{2}=\left\{\left({ }^{\prime \prime} z, z_{n-1}, z_{n}\right) \in \mathbb{C}^{n}\right.$ : $\left.\left.\left.\right|^{\prime \prime} z\right|^{2}+\left|z_{n-1}\right|+\left|z_{n}\right|^{2}<1\right\}$ and $\Sigma$ is biholomorphic to the unit ball $\mathbb{B}$ by means of Cayley's transformation. The mapping ( $\left.{ }^{\prime \prime} z, z_{n-1}, z_{n}\right) \mapsto\left({ }^{\prime \prime} z, z_{n-1}^{2}, z_{n}\right)$ is proper from $\mathbb{B}$ onto $E_{2}$. Thus there exists a proper holomorphic mapping from $\Sigma$ onto $\widehat{G}$ which is not biholomorphic. So Proposition 2 implies that there is no proper holomorphic mapping from $\widehat{G}$ onto $\Sigma$. This contradicts the fact that $\hat{f}$ is proper. Therefore $q$ is a singular point of $\partial B_{\infty}$. Finally, by using the maximum principle and the irreducibility of $H_{\infty}(n \geq 3)$, we get $f\left(V_{f}\right)=H_{\infty}$. This completes the proof of Proposition 1 .

4. Factorization by automorphisms and proof of Theorem 1. In this section we give the proof of Theorem 1. First of all, we need the following lemma to prove that a proper holomorphic self-mapping of $B_{\infty}$ is factored by automorphisms.

Lemma 6. For $n \geq 3, \pi_{1}\left(B_{\infty} \backslash H_{\infty}\right)=\mathbb{Z}$.

Proof. The function $z \mapsto z /\left(1+N_{\infty}(z)\right)$ maps homeomorphically $\mathbb{C}^{n}$ onto $B_{\infty}$ and $H$ onto $H_{\infty}$. Thus $\mathbb{C}^{n} \backslash H$ is homeomorphic to $B_{\infty} \backslash H_{\infty}$. Since $\mathbb{C}^{n} \backslash H$ retracts by deformation onto $S^{2 n-1} \backslash\left(H \cap S^{2 n-1}\right)$, we have $\pi_{1}\left(\mathbb{C}^{n} \backslash H\right)=\pi_{1}\left(S^{2 n-1} \backslash K\right)$ with $K=H \cap S^{2 n-1}$. The mapping

$$
S^{2 n-1} \backslash K \rightarrow S^{1}, \quad x \mapsto \frac{g(x)}{|g(x)|},
$$

$\left(g(x)=\sum_{1 \leq j \leq n} x_{j}^{2}\right)$ is a fibration. Its fiber $F$ is $(n-2-s)$-connected, (i.e. $\pi_{i}(F)=\{0\}$ for all $0 \leq i \leq n-2-s$ ) where $s=\operatorname{dim} H_{\text {sing }}$ (see [8]). Since $s=0$ ( $H$ has only one singularity at 0 ), for $n \geq 3$ we obtain 
$\pi_{1}(F)=\pi_{0}(F)=\{0\}$. The homotopy sequence

$$
\pi_{1}(F) \rightarrow \pi_{1}\left(S^{2 n-1} \backslash K\right) \rightarrow \pi_{1}\left(S^{1}\right) \rightarrow \pi_{0}(F)
$$

is exact; consequently, $\pi_{1}\left(S^{2 n-1} \backslash K\right)=\pi_{1}\left(S^{1}\right)=\mathbb{Z}$.

Proof of Theorem 1. Let $f$ be a proper holomorphic self-mapping of $B_{\infty}$ with branch locus $V_{f}$. We denote by $f^{2}$ the mapping $f \circ f$ and by $V_{f^{2}}$ its branch locus. Assume that $V_{f}$ is not empty. In view of Proposition 1 , Lemma 6 and Theorem 2 the mapping $f$ is factored by a finite subgroup $\Gamma$. In particular we have $f^{-1} \circ f\left(V_{f}\right)=V_{f}$. Then Proposition 1 implies that

$$
f^{-1}\left(H_{\infty}\right)=V_{f}
$$

Since $V_{f^{2}}=V_{f} \cup f^{-1}\left(V_{f}\right)$ and again using Proposition 1 (applied to $f$ and $f^{2}$ ) one has $H_{\infty}=f\left(H_{\infty}\right) \cup H_{\infty}$. It follows from the irreducibility of $H_{\infty}$ that

$$
f\left(H_{\infty}\right)=H_{\infty}
$$

As the automorphism group of $B_{\infty}$ is $S^{1} . O(n, \mathbb{R})$, the elements of $\Gamma$ stabilize $H_{\infty}$. So in view of (7) and the factorization of $f$ we have

$$
f^{-1}\left(H_{\infty}\right)=H_{\infty}
$$

From (6) and (8) we conclude that $V_{f}=H_{\infty}$. But the factorization theorem implies that there exists $\gamma \in \Gamma$ such that $\{\gamma(z)=z\}=H_{\infty}$. This is impossible, since $H_{\infty}$ has a singularity at 0 . This contradiction shows that $V_{f}$ is empty. As the domain $B_{\infty}$ is simply connected, we conclude that $f$ is a biholomorphism.

Remarks. We can repeat the same argument used in the proof of Proposition 1 (second case) to show that there is no proper holomorphic mapping from the minimal ball onto a strongly pseudoconvex bounded domain in $\mathbb{C}^{n}$ with $C^{2}$ boundary. The problem of existence of proper holomorphic mappings from a strongly pseudoconvex bounded domain in $\mathbb{C}^{n}(n \geq 3)$ with $C^{2}$ boundary onto the minimal ball was answered in the negative in [11]. These results solve a question raised by Hahn and Pflug regarding the existence of proper holomorphic mappings between the Euclidean ball and the minimal ball, in a more general context. Note that this question was solved earlier by Oeljeklaus and Youssfi [9] in the case of the Euclidean ball.

The author is grateful to the referee for his encouragement and for his useful remarks on this material.

\section{References}

[1] E. Bedford and S. Bell, Boundary behavior of proper holomorphic correspondences, Math. Ann. 272 (1985), 505-518. 
[2] F. Berteloot and S. Pinchuk, Proper holomorphic mapping between bounded Reinhardt domains in $\mathbb{C}^{2}$, Math Z. 219 (1995), 343-356.

[3] C. Godbillon, Éléments de topologie algébrique, Hermann, Paris, 1971.

[4] K. T. Hahn and P. Pflug, On a minimal complex norm that extends the real Euclidean norm, Monatsh. Math. 105 (1988), 107-112.

[5] R. C. Gunning and H. Rossi, Analytic Functions of Several Complex Variables, Prentice-Hall, 1965.

[6] G. Henkin and A. Iordan, Compactness of the Neumann operator for hyperconvex domains with non-smooth B-regular boundary, Math. Ann. 307 (1997), 151-168.

[7] K. T. Kim, Automorphism groups of certain domains in $\mathbb{C}^{n}$ with singular boundary, Pacific J. Math. 151 (1991), 54-64.

[8] J. W. Milnor, Singular Points of Complex Hypersurfaces, Ann. of Math. Stud. 61, Princeton Univ. Press, 1968.

[9] K. Oeljeklaus and E. H. Youssfi, Proper holomorphic mappings and related automorphism groups, J. Geom. Anal. 7 (1997), 623-636.

[10] K. Oeljeklaus, P. Pflug and E. H. Youssfi, The Bergman kernel of the minimal ball and applications, Ann. Inst. Fourier (Grenoble) 47 (1997), 915-928.

[11] N. Ourimi, Applications holomorphes propres entre certains domaines de $\mathbb{C}^{n}, \mathrm{C} . \mathrm{R}$. Acad. Sci. Paris 326 (1998), 1063-1068.

[12] P. Pflug and E. H. Youssfi, The Lu Qi-Keng conjecture fails for strongly convex algebraic domains, Arch. Math. (Basel) 71 (1998), 240-245.

[13] S. Pinchuk, Holomorphic inequivalence of some classes of domains in $\mathbb{C}^{n}$, Math. USSR Sb. 39 (1981), 61-86.

[14] S. Pinchuk and Sh. I. Tsyganov, The smoothness of CR-mappings between strictly pseudoconvex hypersurfaces, Math. USSR-Izv. 35 (1990), 457-467.

[15] W. Rudin, Proper holomorphic maps and finite reflection groups, Indiana Univ. Math. J. 31 (1992), 701-718.

[16] N. Sibony, Une classe de domaines pseudoconvexes, Duke Math. J. 55 (1987), 299319.

[17] N. Viêt Anh and E. H. Youssfi, Lipschitz estimates for the $\bar{\partial}$-equation on the minimal ball, Michigan Math. J. 49 (2001), 299-323.

ICTP

Math. Section

Strada Costiera, II

34014 Trieste, Italy
Current address:

Faculté des sciences de Bizerte 7021 Jarzouna, Tunisie E-mail: ourimin@yahoo.com

Reçu par la Rédaction le 14.7.2000

Révisé le 9.6.2001 et 9.2.2002 